\title{
GLOBAL STABILITY OF A REACTION-DIFFUSION SYSTEM OF A COMPETITOR-COMPETITOR-MUTUALIST MODEL
}

\author{
Shihe Xu
}

\begin{abstract}
In this paper, we study a reaction-diffusion system of a competitorcompetitor-mutualist model with Neumann boundary condition. Using iteration method, we investigate the global asymptotic stability of the unique positive constant steady-state solution under some assumptions. We also give some sufficient conditions under which there are no nonconstant positive steady-state solution exist.
\end{abstract}

\section{INTRODUCTION}

In this paper, we investigate the global asymptotic stability of a competitorcompetitor-mutualist model as follows:

$$
\left\{\begin{array}{l}
u_{t}-d_{1} \Delta u=\alpha u\left(1-\frac{u}{K_{1}}-\frac{\beta v}{1+m \omega}\right),(t>0, x \in \Omega), \\
v_{t}-d_{2} \Delta v=\delta v\left(1-\frac{v}{K_{2}}\right)-\eta u v,(t>0, x \in \Omega), \\
\omega_{t}-d_{3} \Delta \omega=\gamma \omega\left(1-\frac{\omega}{L_{0}+l u}\right),(t>0, x \in \Omega), \\
\frac{\partial u}{\partial v}=\frac{\partial v}{\partial v}=\frac{\partial \omega}{\partial v}=0,(t>0, x \in \partial \Omega) . \\
u(x, 0)=u_{0}(x), v(x, 0)=v_{0}(x), \omega(x, 0)=\omega_{0}(x) .
\end{array}\right.
$$

Here $u(t, x), v(t, x), \omega(t, x)$ represent the population of two competitor and mutualist with diffusion constants $d_{1}, d_{2}$ and $d_{3}$, respectively, $\Omega$ is a bounded domain in $R^{n}, \frac{\partial}{\partial v}$ is the outer normal derivatives on $\partial \Omega$. The all parameters in (1.1) are positive constants, for details see [8].

Received September 2, 2009, accepted March 23, 2010.

Communicated by J. S. Guo.

2000 Mathematics Subject Classification: 35K57, 92D25.

Key words and phrases: Competitor-competitor-mutualist model, Positive constant steady-state solution, Reaction-diffusion system, Global asymptotic stability.

This work is partially supported by the Natural National Science Foundation of China (No. 10771223, 10926128) and Natural Science Foundation of Guangdong Province (No. 9251064101000015). 
The model was initiated proposed and studied by Rai, Freedman and Addicott in [4] in the ODE form:

$$
\left\{\begin{array}{l}
u_{t}=\alpha u\left(1-\frac{u}{K_{1}}-\beta \frac{v}{1+m \omega}\right),(t>0), \\
v_{t}=\delta v\left(1-\frac{v}{K_{2}}\right)-\eta u v,(t>0), \\
\omega_{t}=\gamma \omega\left(1-\frac{\omega}{L_{0}+l u}\right),(t>0), \\
u(t)=u_{0}, v(x, 0)=v_{0}, \omega(x, 0)=\omega_{0} .
\end{array}\right.
$$

Their model was extended by Zheng [8] to the system (1.1). In [8], under some assumptions the local stability of the unique positive constant steady-state solution is discussed by the method of spectral analysis of linearized operator. The problem (1.2) has been extended to periodic systems by several workers (cf.[1,2,5,7]), where the diffusion coefficients $d_{i}$ and the various reaction rates $\alpha, \beta$, etc. are periodic functions of $t$. Both the existence and asymptotic behavior of time-periodic solutions were investigated in the above papers.

To investigate the asymptotic behavior of the solution of (1.1), as well as the nonexistence of nonconstant positive steady states of (1.1), we also should consider the corresponding steady-state system

$$
\left\{\begin{array}{l}
-d_{1} \Delta u=\alpha u\left(1-\frac{u}{K_{1}}-\frac{\beta v}{1+m \omega}\right),(x \in \Omega), \\
-d_{2} \Delta v=\delta v\left(1-\frac{v}{K_{2}}\right)-\eta u v,(x \in \Omega), \\
-d_{3} \Delta \omega=\gamma \omega\left(1-\frac{\omega}{L_{0}+l u}\right),(x \in \Omega), \\
\frac{\partial u}{\partial v}=\frac{\partial v}{\partial v}=\frac{\partial \omega}{\partial v}=0,(x \in \partial \Omega) .
\end{array}\right.
$$

The main purpose of this paper is to prove the global asymptotic stability of the unique positive constant steady-state solution of (1.3)(see Theorem 2.1) and the nonexistence of nonconstant positive steady-state solutions under some assumptions (see Theorem 2.2).

\section{The Main Result}

Before giving our main results, we recall some results in [8](cf.[4]) which we will use later in the proof of our results.

Theorem A. (1) If $1+m L_{0}=\beta K_{2}$ and $\eta K_{1}<\delta$, then system (1.1) has a unique positive constant steady-state solution $E^{*}=\left(u^{*}, v^{*}, \omega^{*}\right)$, i.e., solution of

$$
\left\{\begin{array}{l}
1-\frac{u}{K_{1}}-\beta \frac{v}{1+m \omega}=0, \\
\delta\left(1-\frac{v}{K_{2}}\right)-\eta u=0, \\
1-\frac{\omega}{L_{0}+l u}=0
\end{array}\right.
$$


and is given by

$$
u^{*}=K_{1}-\frac{\left(\delta-\eta K_{1}\right) \beta K_{2}}{m l \delta}, v^{*}=K_{2}-\frac{\eta}{\delta} u^{*}, \omega^{*}=L_{0}+l u^{*} .
$$

(2) If $1+m L_{0}>\beta K_{2}$ then system (1.1) has a unique positive constant steady-state solution $E^{*}=\left(u^{*}, v^{*}, \omega^{*}\right)$ and is given by the positive value of

$$
u^{*}=\frac{\tau \pm \sqrt{\tau^{2}+4 m l \delta^{2} K_{1}\left(1+m L_{0}-\beta K_{2}\right)}}{2 m l \delta}, v^{*}=K_{2}-\frac{\eta}{\delta} u^{*}, \omega^{*}=L_{0}+l u^{*} .
$$

where

$$
\tau=m l \delta K_{1}+\beta \eta K_{1} K_{2}-\delta\left(1+m L_{0}\right)
$$

provided $u^{*}<\frac{\delta}{\eta}$.

Then our main results are the following Theorems:

Theorem 2.1 Let $E^{*}$ be the unique equilibrium of (1.1) stated in Theorem A. If $1+m L_{0} \geq \beta K_{2}$ and $\eta K_{1}<\delta$, then $E^{*}$ is globally stable, i.e.,

$$
\lim _{t \rightarrow \infty} U(x, t)=E^{*}
$$

for any solution $U(x, t):=(u(x, t), v(x, t), w(x, t))$ of $(1.1)$ with $\left(u_{0}(x), v_{0}(x)\right.$, $\left.w_{0}(x)\right) \not \equiv(0,0,0)$, where $u_{0}, v_{0}, w_{0}$ are nonnegative functions.

Theorem 2.2 Let $\mu_{1}$ be the smallest eigenvalue of the operator $-\Delta$ (except 0$)$ on $\Omega$ with the homogenous Neumann boundary condition and denote $\alpha, \delta, \gamma, \beta, L_{0}, l, \eta, K_{1}, K_{2}, m$ collectively by $\Lambda$ for notational convenience. Then

(i) there exists a positive constant $d=d(n, \Omega, \Lambda)$, such that (1.3) has no nonconstant positive classical solution for $d_{2}, d_{3} \geq d$ provided that $\mu_{1} d_{1}>\delta$,

(ii) there exists a positive constant $d=d(n, \Omega, \Lambda)$, such that (1.3) has no nonconstant positive classical solution for $d_{1}, d_{3} \geq d$ provided that $\mu_{1} d_{2}>\alpha$,

(iii) there exists a positive constant $d=d(n, \Omega, \Lambda)$, such that (1.3) has no nonconstant positive classical solution for $d_{1}, d_{2} \geq d$ provided that $\mu_{1} d_{3}>\gamma$.

\section{The Proof of the Main Results}

Lemma 3.1 (cf. [6]). Let $f, K$ are positive constants, $T \in[0, \infty)$, and if $w$ satisfies

$$
\left\{\begin{array}{l}
w_{t}-d \Delta w \leq(\geq) f w(K-w), \quad(x, t) \in \Omega \times(T, \infty), \\
\frac{\partial w}{\partial \nu}=0, \quad(x, t) \in \partial \Omega \times[T, \infty)
\end{array}\right.
$$

then 


$$
\limsup _{t \rightarrow \infty} \max _{\bar{\Omega}} w(., t) \leq K\left(\liminf _{t \rightarrow \infty} \min _{\bar{\Omega}} w(x, t) \geq K\right)
$$

Lemma 3.2 Let $U(x, t)=(u(x, t), v(x, t), w(x, t))$ be a solution of (1.1), and if $U(x, 0)=\left(u_{0}(x), v_{0}(x), w_{0}(x)\right) \geq(0,0,0), x \in \bar{\Omega}$, then

$$
(0,0,0) \leq \limsup _{t \rightarrow \infty} \max _{\bar{\Omega}} U(x, t) \leq\left(K_{1}, K_{2}, L_{0}+l K_{1}\right), \text { for } t>0, x \in \bar{\Omega} .
$$

Moreover iffor $x \in \bar{\Omega}, U(x, 0) \not \equiv(0,0,0)$, then $U(x, t)>(0,0,0)$ for $x \in \bar{\Omega}, t>0$.

Proof. The first part easily obtain by Lemma 3.1 and if $x \in \bar{\Omega}, U(x, 0) \not \equiv$ $(0,0,0)$, by maximum principle, we can get $U(x, t)>(0,0,0)$.

Lemma 3.3 For any positive classical solution $(u(x), v(x), w(x))$ of (1.3) the following estimates hold

$$
(u(x), v(x), w(x)) \leq\left(K_{1}, K_{2}, L_{0}+l K_{1}\right), \text { for } x \in \bar{\Omega} .
$$

To prove Lemma 3.3, we should use the following Proposition from [3].

Proposition 3.4. Suppose that $g \in(\bar{\Omega} \times \mathbb{R})$, then the following results hold:

(i) If $w \in C^{2}(\Omega) \cap C^{1}(\bar{\Omega})$ satisfies $\Delta w+g(x, w(x)) \geq 0$ for $x \in \Omega$, $\frac{\partial w}{\partial \nu} \geq 0$ for $x \in \partial \Omega$ and $w\left(x_{0}\right)=\max _{\bar{\Omega}} w$, then $g\left(x_{0}, w\left(x_{0}\right)\right) \geq 0$.

(ii) If $w \in C^{2}(\Omega) \cap C^{1}(\bar{\Omega})$ satisfies $\Delta w+g(x, w(x)) \leq 0$ for $x \in \Omega$, $\frac{\partial w}{\partial \nu} \leq 0$ for $x \in \partial \Omega$ and $w\left(x_{0}\right)=\min _{\bar{\Omega}} w$, then $g\left(x_{0}, w\left(x_{0}\right)\right) \leq 0$.

Proof of Lemma 3.3 Let $\phi=d_{1} u$. From the first equation of (1.3) we have

$$
-\Delta \phi=\alpha u\left(1-\frac{u}{K_{1}}-\frac{\beta v}{1+m \omega}\right),(x \in \Omega) ; \frac{\partial \phi}{\partial \nu}=0,(x \in \partial \Omega) .
$$

Let $x_{0} \in \bar{\Omega}$ be such that $\phi\left(x_{0}\right)=\max _{\bar{\Omega}} \phi$. Then by proposition 3.4 and the positiveness of $u, v, w$, we get $1-\frac{u\left(x_{0}\right)}{K_{1}}-\frac{\beta v\left(x_{0}\right)}{1+m \omega\left(x_{0}\right)} \geq 0$. Hence $u\left(x_{0}\right) \leq K_{1}$ which in turn implies that $\max _{\bar{\Omega}} u \leq K_{1}$.

Analogously, by setting $V=d_{2} v$ and $W=d_{3} w$, we can easily get from proposition 3.4 that

$$
v\left(x_{0}\right) \leq K_{2}, w\left(x_{0}\right) \leq L_{0}+l K_{1} .
$$

These inequalities certainly implies our assertion.

Proof of Theorem 2.1. We first prove if $1+m L_{0}>\beta K_{2}$ and $\eta K_{1}<\delta$ hold, then $E^{*}$ is globally stable. Since $U(x, t)=(u(x, t), v(x, t), w(x, t))$ is a solution of (1.1), by Lemma 3.2, 


$$
\begin{aligned}
& u_{t}-d_{1} \Delta u \leq \alpha u\left(1-\frac{u}{K_{1}}\right) \\
& v_{t}-d_{2} \Delta v \leq v\left(1-\frac{v}{K_{2}}\right), \\
& \omega_{t}-d_{3} \Delta \omega \geq \gamma \omega\left(1-\frac{\omega}{L_{0}}\right) .
\end{aligned}
$$

By Lemma 3.1, we have

$$
\begin{aligned}
& \lim \sup _{t \rightarrow \infty} \max _{\bar{\Omega}} u(., t) \leq K_{1}=: \bar{u}_{1}, \\
& \lim \sup _{t \rightarrow \infty} \max _{\bar{\Omega}} v(., t) \leq K_{2}=: \bar{v}_{1}, \\
& \lim \sup _{t \rightarrow \infty} \max _{\bar{\Omega}} \omega(., t) \geq L_{0}=: \underline{\omega}_{1} .
\end{aligned}
$$

For any given $\varepsilon>0$, there exists $T_{1}^{\varepsilon}$ sufficient large, such that

$$
\begin{aligned}
& u(x, t)<\bar{u}_{1}+\varepsilon, \text { for } x \in \bar{\Omega}, t>T_{1}^{\epsilon}, \\
& v(x, t)<\bar{v}_{1}+\varepsilon, \text { for } x \in \bar{\Omega}, t>T_{1}^{\epsilon}, \\
& \omega(x, t)>\underline{\omega}_{1}+\varepsilon, \text { for } x \in \bar{\Omega}, t>T_{1}^{\epsilon} .
\end{aligned}
$$

By the second and third equations of (1.1), we have for $x \in \Omega, t>T_{1}^{\epsilon}$,

$$
\begin{gathered}
v_{t}-d_{2} \Delta v \geq \delta v\left(1-\frac{v}{K_{2}}\right)-\eta\left(\bar{u}_{1}+\varepsilon\right) v, \\
\omega_{t}-d_{3} \Delta \omega \leq \gamma \omega\left(1-\frac{\omega}{L_{0}+l\left(\bar{u}_{1}+\varepsilon\right)}\right),
\end{gathered}
$$

then again by Lemma 3.1, we obtain

$$
\begin{gathered}
\liminf _{t \rightarrow \infty} \min _{\bar{\Omega}} v(x, t) \geq\left[1-\frac{\eta}{\delta}\left(\bar{u}_{1}+\varepsilon\right)\right] K_{2}, \\
\limsup _{t \rightarrow \infty} \max _{\bar{\Omega}} \omega(., t) \leq L_{0}+l\left(\bar{u}_{1}+\varepsilon\right) .
\end{gathered}
$$

By the arbitrariness of $\varepsilon>0$, it follows that

$$
\begin{gathered}
\liminf _{t \rightarrow \infty} \min _{\bar{\Omega}} v(x, t) \geq\left(1-\frac{\eta}{\delta} \bar{u}_{1}\right) K_{2}=: \underline{v}_{1}, \\
\limsup _{t \rightarrow \infty} \max _{\bar{\Omega}} \omega(., t) \leq L_{0}+l \bar{u}_{1}=: \bar{\omega}_{1} .
\end{gathered}
$$

Hence, there exists $T_{2}^{\varepsilon}$ sufficient large, such that

$$
\omega(x, t) \leq \bar{\omega}_{1}+\varepsilon \text { for } x \in \bar{\Omega}, t \geq T_{2}^{\epsilon} .
$$

From the equation for $u$ in (1.1) 


$$
u_{t}-d_{1} \Delta u \geq \alpha u\left(1-\frac{u}{K_{1}}-\frac{\beta \bar{v}_{1}}{1+m\left(\underline{\omega}_{1}+\varepsilon\right)}\right) .
$$

Thanks to Lemma 3.1

$$
\liminf _{t \rightarrow \infty} \min _{\bar{\Omega}} u(., t) \geq\left(1-\frac{\beta \bar{v}_{1}}{1+m\left(\underline{\omega}_{1}+\varepsilon\right)}\right) K_{1} .
$$

By the arbitrariness of $\varepsilon>0$, it follows that

$$
\liminf _{t \rightarrow \infty} \min _{\bar{\Omega}} u(., t) \geq\left(1-\frac{\beta \bar{v}_{1}}{1+m \underline{\omega}_{1}}\right) K_{1}=: \underline{u}_{1} .
$$

Hence, for any $0<\varepsilon<\bar{u}_{1}$ there exists $T_{3}^{\varepsilon}$ sufficient large, such that

$$
u(x, t) \geq \underline{u}_{1}-\varepsilon \text { for } x \in \bar{\Omega}, t \geq T_{3}^{\epsilon} .
$$

From the equation for $\omega$ and $v$ in (1.1)

$$
\begin{gathered}
\omega_{t}-d_{3} \Delta \omega \geq \gamma \omega\left(1-\frac{\omega}{L_{0}+l\left(\underline{u}_{1}-\varepsilon\right)}\right), \\
v_{t}-d_{2} \Delta v \leq \alpha v\left(1-\frac{v}{K_{2}}-\frac{\eta}{\delta}\left(\underline{u}_{1}-\varepsilon\right)\right) .
\end{gathered}
$$

Thanks to Lemma 3.1

$$
\begin{gathered}
\liminf _{t \rightarrow \infty} \min _{\bar{\Omega}} \omega(., t) \geq L_{0}+l\left(\underline{u}_{1}-\varepsilon\right), \\
\limsup _{t \rightarrow \infty} \max _{\bar{\Omega}} v(., t) \leq\left(1-\frac{\eta}{\delta}\left(\underline{u}_{1}-\varepsilon\right)\right) K_{2} .
\end{gathered}
$$

By the arbitrariness of $\varepsilon>0$, it follows that

$$
\begin{gathered}
\liminf _{t \rightarrow \infty} \min _{\bar{\Omega}} \omega(., t) \geq L_{0}+l \underline{u}_{1}=: \underline{\omega}_{2}, \\
\limsup _{t \rightarrow \infty} \max _{\bar{\Omega}} v(., t) \leq\left(1-\frac{\eta}{\delta} \underline{u}_{1}\right) K_{2}=: \bar{v}_{2} .
\end{gathered}
$$

Then there exists $T_{4}^{\varepsilon}$ sufficiently large, for $x \in \bar{\Omega}, t \geq T_{4}^{\epsilon}$ such that

$$
\begin{aligned}
& \omega(x, t) \geq \underline{\omega}_{2}-\varepsilon, \\
& v(x, t) \leq \bar{v}_{2}+\varepsilon .
\end{aligned}
$$

From the equation for $u$ in (1.1)

$$
u_{t}-d_{1} \Delta u \geq \alpha u\left(1-\frac{u}{K_{1}}-\frac{\beta\left(\bar{v}_{2}+\varepsilon\right)}{1+m\left(\underline{\omega}_{2}-\varepsilon\right)}\right) .
$$

Thanks to Lemma 3.1 and by the arbitrariness of $\varepsilon>0$, it follows that

$$
\liminf _{t \rightarrow \infty} \min _{\bar{\Omega}} u(., t) \geq\left(1-\frac{\beta \bar{v}_{2}}{1+m \underline{\omega}_{2}}\right) K_{1}=: \underline{u}_{2} .
$$


Applying the inductive method we can construct sequences $\left\{\bar{u}_{i}\right\},\left\{\bar{v}_{i}\right\},\left\{\bar{\omega}_{i}\right\},\left\{\underline{u}_{i}\right\}$, $\left\{\underline{v}_{i}\right\},\left\{\underline{\omega}_{i}\right\}$ as follows: $\bar{u}_{1}=K_{1}, \bar{v}_{1}=K_{2}, \bar{\omega}_{1}=L_{0}+l \bar{u}_{1}, \underline{u}_{1}=\left(1-\frac{\beta \bar{v}_{1}}{1+m \underline{\omega}_{1}}\right) K_{1}, \underline{v}_{1}$ $=\left(1-\frac{\eta}{\delta} \bar{u}_{1}\right) K_{2}, \underline{\omega}_{1}=L_{0}$ and for $n>1$

$$
\begin{aligned}
& \bar{v}_{n}=\left(1-\frac{\eta}{\delta} \underline{u}_{n-1}\right) K_{2}, \underline{v}_{n}=\left(1-\frac{\eta}{\delta} \bar{u}_{n}\right) K_{2}, \underline{\omega}_{n}=L_{0}+l \underline{u}_{n-1}, \\
& \bar{\omega}_{n}=L_{0}+l \bar{u}_{n}, \underline{u}_{n}=\left(1-\frac{\beta \bar{v}_{n}}{1+m \underline{\omega}_{n}}\right) K_{1}, \bar{u}_{n}=\left(1-\frac{\beta \underline{v}_{n-1}}{1+m \bar{\omega}_{n-1}}\right) K_{1} .
\end{aligned}
$$

The constants $\bar{u}_{1}, \bar{v}_{1}, \bar{\omega}_{1}, \underline{u}_{2}, \underline{v}_{1}, \underline{\omega}_{1}$ constructed above satisfy the relation:

$$
\begin{aligned}
& \underline{v}_{1} \leq \bar{v}_{1}, \underline{u}_{1} \leq \bar{u}_{1}, \\
& \underline{u}_{1} \leq \lim \inf _{t \rightarrow \infty} \min _{\bar{\Omega}} u(., t) \leq \lim \sup _{t \rightarrow \infty} \max _{\bar{\Omega}} u(., t) \leq \bar{u}_{1}, \\
& \underline{v}_{1} \leq \lim \inf _{t \rightarrow \infty} \min _{\bar{\Omega}} v(., t) \leq \lim \sup _{t \rightarrow \infty} \max _{\bar{\Omega}} v(., t) \leq \bar{v}_{1},
\end{aligned}
$$

and the sequence $\left\{\bar{u}_{i}\right\},\left\{\bar{v}_{i}\right\},\left\{\bar{\omega}_{i}\right\},\left\{\underline{u}_{i}\right\},\left\{\underline{v}_{i}\right\},\left\{\underline{\omega}_{i}\right\}$ satisfy

$$
\begin{aligned}
& \underline{u}_{i} \leq \liminf _{t \rightarrow \infty} \min _{\bar{\Omega}} u(., t) \leq \lim \sup _{t \rightarrow \infty} \max _{\bar{\Omega}} u(., t) \leq \bar{u}_{i}, \\
& \underline{v}_{i} \leq \lim \inf _{t \rightarrow \infty} \min _{\bar{\Omega}} v(., t) \leq \lim \sup _{t \rightarrow \infty} \max _{\bar{\Omega}} v(., t) \leq \bar{v}_{i}, \\
& 0<\underline{u}_{1} \leq \underline{u}_{2} \leq \ldots \leq \underline{u}_{n-1} \leq \underline{u}_{n} \leq \bar{u}_{n} \leq \bar{u}_{n-1} \leq \ldots \leq \bar{u}_{2} \leq \bar{u}_{1}=K_{1}, \\
& 0<\underline{v}_{1} \leq \underline{v}_{2} \leq \ldots \leq \underline{v}_{n-1} \leq \underline{v}_{n} \leq \bar{v}_{n} \leq \bar{v}_{n-1} \leq \ldots \leq \bar{v}_{2} \leq \bar{v}_{1}=K_{2}, \\
& L_{0}<\underline{\omega}_{1} \leq \underline{\omega}_{2} \leq \ldots \leq \underline{\omega}_{n-1} \leq \underline{\omega}_{n} \leq \bar{\omega}_{n} \leq \bar{\omega}_{n-1} \leq \ldots \leq \bar{\omega}_{2} \leq \bar{\omega}_{1}=L_{0}+K_{1} .
\end{aligned}
$$

Actually, since $1+m L_{0}>\beta K_{2}$, we have

$$
K_{1}=\bar{u}_{1} \geq \underline{u}_{1}=\left(1-\frac{\beta \bar{v}_{1}}{1+m \underline{\omega}_{1}}\right) K_{1}=\left(1-\frac{\beta K_{2}}{1+m L_{0}}\right) K_{1}>0 .
$$

Then noting that $\eta K_{1}<\delta$, we obtain

$$
\begin{gathered}
\bar{v}_{2}=\left(1-\frac{\eta}{\delta} \underline{u}_{1}\right) K_{2} \geq \underline{v}_{1}=\left(1-\frac{\eta}{\delta} \bar{u}_{1}\right) K_{2}>0, \\
L_{0}<\underline{\omega}_{2}=L_{0}+l \underline{u}_{1} \leq \bar{\omega}_{1}=L_{0}+l \bar{u}_{1} .
\end{gathered}
$$

Thus

$$
\underline{u}_{2}=\left(1-\frac{\beta \bar{v}_{2}}{1+m \underline{\omega}_{2}}\right) K_{1} \leq \bar{u}_{2}=\left(1-\frac{\beta \underline{v}_{1}}{1+m \bar{\omega}_{1}}\right) K_{1} \leq \bar{u}_{1},
$$

and

$$
\underline{u}_{2}=\left(1-\frac{\beta \bar{v}_{2}}{1+m \underline{\omega}_{2}}\right) K_{1} \geq \underline{u}_{1}=\left(1-\frac{\beta \bar{v}_{1}}{1+m \underline{\omega}_{1}}\right) K_{1}>0 .
$$

The above conclusions show that

$$
0<\underline{u}_{1} \leq \underline{u}_{2} \leq \bar{u}_{2} \leq \bar{u}_{1}=K_{1} .
$$


Use induction principle, it is not hard to prove that

$$
0<\underline{u}_{1} \leq \underline{u}_{2} \leq \ldots \leq \underline{u}_{n-1} \leq \underline{u}_{n} \leq \bar{u}_{n} \leq \bar{u}_{n-1} \leq \ldots \leq \bar{u}_{2} \leq \bar{u}_{1}=K_{1} .
$$

Then using inductive principle, the proof of the remainder of (3.6) is immediately get from (3.5) and

$\bar{v}_{n}=\left(1-\frac{\eta}{\delta} \underline{u}_{n-1}\right) K_{2}, \underline{v}_{n}=\left(1-\frac{\eta}{\delta} \bar{u}_{n}\right) K_{2}$ and $\underline{\omega}_{n}=L_{0}+l \underline{u}_{n-1}, \bar{\omega}_{n}=L_{0}+l \bar{u}_{n}$.

By monotone bounds principle, we get

$$
\lim _{n \rightarrow \infty} \bar{u}_{n}=\bar{u}, \lim _{n \rightarrow \infty} \underline{u}_{n}=\underline{u} .
$$

In (3.4), letting $n \rightarrow \infty$, we have

$$
\begin{gathered}
\bar{v}=\left(1-\frac{\eta}{\delta} \underline{u}\right) K_{2}, \underline{v}=\left(1-\frac{\eta}{\delta} \bar{u}\right) K_{2}, \\
\underline{\omega}=L_{0}+l \underline{u}, \bar{\omega}=L_{0}+l \bar{u}, \\
\underline{u}=\left(1-\frac{\beta \bar{v}}{1+m \underline{\omega}}\right) K_{1}, \bar{u}=\left(1-\frac{\beta \underline{v}}{1+m \bar{\omega}}\right) K_{1} .
\end{gathered}
$$

It follows from (3.7) (3.8) that

$$
\bar{\omega}-\underline{\omega}=l(\bar{u}-\underline{u}), \bar{v}-\underline{v}=\frac{\eta}{\delta}(\bar{u}-\underline{u}) .
$$

Next, we will prove that $\bar{u}=\underline{u}$, and hence $\bar{v}=\underline{v}, \bar{\omega}=\underline{\omega}$ by (3.10). Substituting the first equation of (3.7)and (3.8) to the first equation of (3.9), we get $\underline{u}$ satisfies the following equation:

$$
m l \delta u^{2}-\tau u-\delta K_{1}\left(1+m L_{0}-\beta K_{2}\right)=0,
$$

where

$$
\tau=m l \delta K_{1}+\beta \eta K_{1} K_{2}-\delta\left(1+m L_{0}\right) .
$$

Similarly substituting the second equation of (3.7)and (3.8)to the second equation of (3.9), we get $\bar{u}$ also satisfies the equation (3.11). From [8], we known that under the condition

$$
1+m L_{0}>\beta K_{2} \text { and } \eta K_{1}<\delta,
$$

$E^{*}$ exists uniquely. This implies $\bar{u}=\underline{u}=u^{*}$. Consequently $\bar{v}=\underline{v}=v^{*}, \bar{\omega}=\underline{\omega}=$ $\omega^{*}$. The fact combined with (3.6)implies

$$
\lim _{t \rightarrow \infty} U(x, t)=E^{*}
$$


uniformly on $\bar{\Omega}$. The proof is complete.

Next, we prove that if $1+m L_{0}=\beta K_{2}$ and $\eta K_{1}<\delta$, then $E^{*}$ is globally stable. By Lemma 3.2, for any nonnegative initial function which is not identical to zero the solution of (1.1) is positive. therefore there exists $t^{*}>0, \delta_{i}>0,(i=1,2,3)$ such that

$$
\delta_{i} \leq \min \left\{u(t, x), v(t, x), \omega(t, x) ; t^{*} \leq t \leq t^{*}+\tau, x \in \bar{\Omega}\right\},
$$

then $\left(\delta_{1}, \delta_{2}, \delta_{3}\right) \leq(u(t, x), v(t, x), \omega(t, x)) \leq\left(K_{1}, K_{2}, L_{0}+l K_{1}\right)$ on $\left[t_{1}-\tau, t_{1}\right] \times \bar{\Omega}$ where $t_{1}=t^{*}+\tau$. Using $(u(t, x), v(t, x), \omega(t, x))$ as the initial function in the domain $\left[t_{1}-\tau, \infty\right) \times \Omega$, similar to prove the first part of Theorem 2.1 above, we construct sequences $\left\{\bar{u}_{i}\right\},\left\{\bar{v}_{i}\right\},\left\{\bar{\omega}_{i}\right\},\left\{\underline{u}_{i}\right\},\left\{\underline{v}_{i}\right\},\left\{\underline{\omega}_{i}\right\}$ as follows: $\bar{u}_{1}=(1-$ $\left.\frac{\delta_{2}}{1+m\left(L_{0}+K_{1}\right)}\right) K_{1}, \bar{v}_{1}=\left(1-\frac{\eta}{\delta} \underline{u}_{1}\right) K_{2}, \bar{\omega}_{1}=L_{0}+l \bar{u}_{1}, \underline{u}_{1}=\left(1-\frac{\beta \bar{v}_{1}}{1+m \underline{\omega}_{1}}\right) K_{1}, \underline{v}_{1}=$ $\left(1-\frac{\eta}{\delta} \bar{u}_{1}\right) K_{2}, \underline{\omega}_{1}=L_{0}$ and for $n>1$

$$
\begin{aligned}
& \bar{v}_{n}=\left(1-\frac{\eta}{\delta} \underline{u}_{n-1}\right) K_{2}, \underline{v}_{n}=\left(1-\frac{\eta}{\delta} \bar{u}_{n}\right) K_{2}, \underline{\omega}_{n}=L_{0}+l \underline{u}_{n-1}, \\
& \bar{\omega}_{n}=L_{0}+l \bar{u}_{n}, \underline{u}_{n}=\left(1-\frac{\beta \bar{v}_{n}}{1+m \underline{\omega}_{n}}\right) K_{1}, \bar{u}_{n}=\left(1-\frac{\beta \underline{v}_{n-1}}{1+m \bar{\omega}_{n-1}}\right) K_{1} .
\end{aligned}
$$

Consequently under condition $1+m L_{0}=\beta K_{2}$ and $\eta K_{1}<\delta$ we have $\underline{u}_{1}=$ $\left(1-\frac{\beta \bar{v}_{1}}{1+m \underline{\omega}_{1}}\right) K_{1}>0, \underline{v}_{1}=\left(1-\frac{\eta}{\delta} \bar{u}_{1}\right) K_{2}>0, \underline{\omega}_{1}=L_{0}>0$. By inductive principle, we can get the sequence $\left\{\bar{u}_{i}\right\},\left\{\bar{v}_{i}\right\},\left\{\bar{\omega}_{i}\right\},\left\{\underline{u}_{i}\right\},\left\{\underline{v}_{i}\right\},\left\{\underline{\omega}_{i}\right\}$ satisfy (3.6). Then letting $n \rightarrow \infty$, the following proof is similar to the proof of part one above, we omit it here. The proof of Theorem 2.1 is complete.

Proof of Theorem 2.2. For any $\varphi \in L^{1}(\Omega)$, we write $\bar{\varphi}=\frac{1}{|\Omega|} \int_{\Omega} \varphi d x$. Let $u, v, \omega$ be any positive classical solution of (1.3). Multiplying the corresponding differential equation in (1.3) by $u-\bar{u}, v-\bar{v}, \omega-\bar{\omega}$ respectively, and then integrating over $\Omega$ by part, by the $\varepsilon$ - Young's inequality, we have

$$
\begin{aligned}
& d_{1} \int_{\Omega}|\nabla(u-\bar{u})|^{2} d x \\
= & \int_{\Omega}\left\{\left[\alpha u\left(1-\frac{u}{K_{1}}-\frac{\beta v}{1+m \omega}\right)\right]-\left[\alpha \bar{u}\left(1-\frac{\bar{u}}{K_{1}}-\frac{\beta \bar{v}}{1+m \bar{\omega}}\right)\right]\right\}(u-\bar{u}) d x \\
\leq & \int_{\Omega}\left(\alpha|u-\bar{u}|^{2}+\alpha \beta K_{1}|u-\bar{u}||v-\bar{v}|+\alpha \beta K_{1} K_{2}|u-\bar{u}||\omega-\bar{\omega}|\right) d x \\
\leq & (\alpha+\varepsilon) \int_{\Omega}|u-\bar{u}|^{2} d x+C_{1}(\varepsilon, \Lambda) \int_{\Omega}|v-\bar{v}|^{2}+C_{2}(\varepsilon, \Lambda) \int_{\Omega}|\omega-\bar{\omega}|^{2} d x \\
& d_{2} \int_{\Omega}|\nabla(v-\bar{v})|^{2} d x
\end{aligned}
$$




$$
\begin{aligned}
= & \int_{\Omega}\left\{\left[\delta v\left(1-\frac{v}{K_{2}}-\frac{\eta u}{\delta}\right)\right]-\left[\delta \bar{v}\left(1-\frac{\bar{v}}{K_{2}}-\frac{\eta \bar{u}}{\delta}\right)\right]\right\}(v-\bar{v}) d x \\
\leq & \int_{\Omega}\left(\delta|v-\bar{v}|^{2}+\eta K_{2}|u-\bar{u}||v-\bar{v}| d x\right. \\
\leq & \varepsilon \int_{\Omega}|u-\bar{u}|^{2} d x+C_{3}(\varepsilon, \Lambda) \int_{\Omega}|v-\bar{v}|^{2} d x \\
& d_{3} \int_{\Omega}|\nabla(\omega-\bar{\omega})|^{2} d x \\
= & \int_{\Omega}\left[\gamma \omega\left(1-\frac{\omega}{L_{0}+l u}\right)-\gamma \bar{\omega}\left(1-\frac{\bar{\omega}}{L_{0}+l \bar{u}}\right)\right](\omega-\bar{\omega}) d x \\
\leq & \int_{\Omega}\left(\gamma|\omega-\bar{\omega}|^{2}+\gamma\left(1+\frac{l}{L_{0}} K_{1}\right)^{2}\right) l|u-\bar{u}||\omega-\bar{\omega}| d x \\
\leq & \varepsilon \int_{\Omega}|u-\bar{u}|^{2} d x+C_{4}(\varepsilon, \Lambda) \int_{\Omega}|v-\bar{v}|^{2} d x+C_{5}(\varepsilon, \Lambda) \int_{\Omega}|\omega-\bar{\omega}|^{2} d x .
\end{aligned}
$$

Here, we used Lemma 3.3. Consequently, there exists a sufficient small positive constant $\varepsilon$ which only depends on $\Lambda$ such that

$$
\begin{aligned}
& d_{1} \int_{\Omega}|\nabla(u-\bar{u})|^{2} d x+d_{2} \int_{\Omega}|\nabla(v-\bar{v})|^{2} d x+d_{3} \int_{\Omega}|\nabla(\omega-\bar{\omega})|^{2} d x \\
& \leq(\alpha+\varepsilon) \int_{\Omega}|u-\bar{u}|^{2} d x+C(\varepsilon, \Lambda) \int_{\Omega}|v-\bar{v}|^{2} d x+C(\varepsilon, \Lambda) \int_{\Omega}|v-\bar{v}|^{2} d x .
\end{aligned}
$$

It follows from Poincaré inequality that

$$
\begin{aligned}
& \mu_{1}\left[\int_{\Omega} d_{1}|(u-\bar{u})|^{2} d x+\int_{\Omega} d_{2}|(v-\bar{v})|^{2} d x+\int_{\Omega} d_{3}|(\omega-\bar{\omega})|^{2}\right] d x \\
& \leq(\alpha+\varepsilon) \int_{\Omega}|u-\bar{u}|^{2} d x+C(\varepsilon, \Lambda) \int_{\Omega}|v-\bar{v}|^{2} d x+C(\varepsilon, \Lambda) \int_{\Omega}|v-\bar{v}|^{2} d x .
\end{aligned}
$$

Since $\mu_{1} d_{1}>\alpha$ we may chose $\varepsilon>0$ sufficient small, such that $\mu_{1} d_{1}>\alpha+\varepsilon$. Consequently, by above inequality, we have

$$
\begin{aligned}
& \mu_{1}\left[\int_{\Omega} d_{2}|(v-\bar{v})|^{2} d x+\int_{\Omega} d_{3}|(\omega-\bar{\omega})|^{2}\right] d x \\
\leq & C(\varepsilon, \Lambda) \int_{\Omega}|v-\bar{v}|^{2} d x+C(\varepsilon, \Lambda) \int_{\Omega}|v-\bar{v}|^{2} d x .
\end{aligned}
$$

This implies that $v=\bar{v}=\omega=\bar{\omega}=$ constant, and in turn $u=\bar{u}=$ constant, if $d_{2}, d_{3}>d:=\frac{C(\varepsilon, \Lambda)}{\mu_{1}}$, which asserts our result (i).

The arguments of (ii)(iii) are rather similar to the ones given in the proof of (i), and are thus omitted. 


\section{ACKNOWLEDGMENTS}

The author expresses his thanks to the references for their valuable suggestions on modification of the original manuscript.

\section{REFERENCES}

1. H. Y. Du, Positive periodic solutions of a competitor-competitor-mutualist model, Diff. Inte. Equa., 19 (1996), 1043-1066.

2. M. S. Fu and B. S. Cui, Persistence in a periodic competitor-competitor-mutualist diffusion system, J. Math. Anal. Appl., 263 (2001), 234-245.

3. Y. Lou and W. M. Ni, Diffusion, self diffusion and cross-diffusion, J. Diff. Equa., 131 (1996), 79-131.

4. B. Rai, H. I. Freedman and J. F. Addicott, Analysis of three species models of mutualism in predator-prey and competitive systems, Math. Biosc., 65 (1983), 1350.

5. S. A. Tineo, Asymptotic behavior of solutions of a periodic reaction-diffusion system of a competitor-competitor-mutualist model, J. Diff. Equa., 108 (1994), 326-341.

6. X. M. Wang, Global asymptotic stability of positive steady states of a diffusive ratiodependent prey-predator model, Appl. Math. Lett., 11 (2008), 1215-1220.

7. L. Zhou and Y. P. Fu, Periodic quasimonotone global attractor of nonlinear parabolic systems with discrete delays, J. Math. Anal. Appl., 250 (2000), 139-161.

8. N. S. Zheng, A reaction-diffusion systems of a competitor-competitor-mutualist model, J. Math. Anal. Appl., 124 (1987), 254-280.

\section{Shihe Xu}

Department of Mathematics

Zhaoqing University

Zhaoqing 526061

P. R. China

E-mail: shihexu03@yahoo.com.cn 